\title{
Review
}

\section{Programmed cell death during vascular system formation}

\author{
Hiroo Fukuda \\ Botanical Gardens, Faculty of Science, University of Tokyo, Tokyo 112, Japan \\ tel: 81-3-5684-8597; fax: 81-3-3814-0139; \\ email: sfukuda@hongo.ecc.u-tokyo.ac.jp
}

Received 28.4.97; revised 3.7.97; accepted 14.7.97

Edited by B.A. Osborne

\begin{abstract}
Vascular plants have vessels and tracheids composed of dead tracheary elements. Differentiation of procambial or cambial cells to tracheary elements is a typical example of programmed cell death in higher plants. Recent studies on tracheary element differentiation, in particular with an in vitro differentiation system, have revealed a unique cell death process which differs from apoptosis. Herein I summarize the present knowledge about the induction of cell death, the morphological features of cell death, and the mechanism of autolysis, including the involvement of a DNase and cysteine proteases, during tracheary element differentiation.
\end{abstract}

Keywords: apoptosis; cysteine protease; DNase; necrosis; programmed cell death; tracheary element differentiation

Abbreviations: TE, tracheary element

\section{Introduction}

In higher plants as well as in animals, cell death is programmed in various processes of development (Jones and Dangl, 1996). Vessels and tracheids, which are conductive tissues in vascular plants, are constructed from aligned dead tracheary elements (TEs). TEs are differentiated from procambial cells in the primary xylem and from cells produced by the vascular cambium in the secondary xylem. At maturity, TEs lose their nuclei and cell contents, leaving hollow dead cells through which water and nutrients pass. The final stage of TE differentiation involves cell death and, therefore, this differentiation focuses our attention on a typical example of programmed cell death in higher plants (Fukuda, 1996, 1997). TE differentiation is rather easily induced in vitro, and therefore many TE-inductive systems have been established which have contributed to increases in the understandings of the cell death process (Fukuda, 1996). In particular, a Zinnia experimental system in which single mesophyll cells transdifferentiate synchronously into TEs has proved to be extremely useful for studies of the sequence of events in TE differentiation (Fukuda and Komamine, 1980a,b; Chasan, 1994; Fukuda, 1997). Detailed analyses of this transdifferentiation have provided a number of cytological, biochemical, and molecular markers that should be useful for understanding the cell death process. In this article, I review the present knowledge of programmed cell death during TE differentiation, based on recent results obtained mainly from the Zinnia system.

\section{Sequence of TE differentiation}

Transdifferentiation of parenchyma cells into TEs can occur directly without intervening cell cycling (Fukuda and Komamine, 1980b). Fukuda $(1996,1997)$ have revealed that the transdifferentiation of parenchyma cells into TEs is divided into three stages, I, II and III, based on results from the in vitro differentiation system of Zinnia elegans (Figure 1). Isolated single mesophyll cells of Zinnia become committed to transdifferentiation into TEs by phytohormones, a combination of auxin and cytokinin. Transdifferentiation after the commitment involves three stages. Stage I, which immediately follows the induction of differentiation by phytohormones and wounding, corresponds to a functional dedifferentiation process during which isolated mesophyll cells lose their potential to function as photosynthetic cells. This process is not accompanied by cell division. The functional dedifferentiation is typical in that the reticulate arrays of actin filaments over chloroplasts, which may anchor chloroplasts to the plasma membrane, become a three-dimensional network throughout the entire length of the cell (Kobayashi et al, 1987), causing chloroplasts to leave the vicinity of the plasma membrane and the mesophyll cells to lose their photosynthetic function.

During Stage II, various vascular cell-specific genes such as TED2, TED3 and TED4 begin to be expressed (Demura and Fukuda, 1993, 1994). The analysis of Stage II with these genes as markers have suggested that, during Stage II, dedifferentiated cells differentiate into TE precursor cells, corresponding to the process of differentiation from meristematic cells to TE precursor cells via procambial initials in situ (Fukuda, 1994).

Stage III, final stage of differentiation involves secondary wall formation and cell death, which are common both in vitro and in situ. During Stage III, various enzymes and structural proteins associated with the secondary wall formation and cell death are expressed. The secondary wall formation and cell death seem to be tightly coupled (Fukuda, 1997).

\section{Induction of entry into stage III and of cell death}

Toxins, high concentrations of salts, and some chemicals can induce apoptosis-like death of particular cells in plants (Wang et al, 1996a; Ryerson and Heath, 1996; Katsuhara and 
Kawasaki, 1996). Phytohormones sometimes function in the induction of developmentally programmed cell death. For example, the death of aleurone cells is induced by gibberellic acid (GA) and suppressed by abscisic acid (ABA) (Kuo et al, 1996; Wang et al, 1996b). In contrast, GA inhibits the cell death process in pea ovary senescence (Vercher et al, 1987). However, inducers for cell death during most of the developmental processes in plants have not been identified.

In TE differentiation, the transition from Stage II to Stage III may be an irreversible checkpoint of differentiation (Figure 1). The addition of cycloheximide and actinomycin $D$ until the time of the transition inhibited TE differentiation (Fukuda and Komamine, 1983). CaM antagonists specifically prevented cultured Zinnia mesophyll cells from entering Stage III (Kobayashi and Fukuda, 1994). Before entering Stage III, CaM levels increase transiently, subsequently, a few CaM-binding proteins start to be expressed in a differentiation-specific manner (Kobayashi and Fukuda, 1994). Membrane-associated calcium ions were higher in TE precursor cells than in control cells of Zinnia (Roberts and Haigler, 1989). Therefore, the calcium/ CaM system may be involved in the entry into Stage III.

This entry also seems to be regulated by endogenous brassinosteroids. The inhibition of brassinosteroid biosynthesis by a plant-growth retardant, uniconazol, severely prevented TE differentiation from Zinnia mesophyll cells (Iwasaki and Shibaoka, 1991). Uniconazol did not inhibit the expression of genes that were expressed at Stage I or Stage II, such as genes for PR protein and TEDs. However, it did specifically prevent the expression of Stage III-specific genes, including genes both for enzymes involved in the secondary wall formation (phenylalanine ammonia-lyases and cinnamic acid 4-hydroxylase etc.) and for enzymes involved in cell death (a cysteine protease etc.) (Yamamoto et al, 1997). The inhibition of both TE differentiation and gene expression was recovered by supplying brassinolide, an active brassinosteroid. Therefore, it is possible that endogenous brassinosteroids function in the entry into Stage III.

\section{The cell death process of TEs compared with apoptosis}

Apoptosis in animals is an active process that involves nuclear shrinkage, cullular shrinkage, membrane bubbling, the formation of apoptotic bodies and digestion by macrophages (Kerr and Harmon, 1991). Characteristic features of DNA degradation such as DNA laddering or TUNEL-positive signal are often used to detect apoptosis. In contrast, necrosis is thought to be a passive cell death process. In necrosis, organelles, especially mitochondria, swell and lysosomes function in their degradation. There have been a number of ultrastructural observations of cell death processes during TE differentiation from procambial cells in situ and parenchyma cells in vitro (O'Brien and Thimann, 1967; Srivastava and Singh, 1972; Lai and Srivastava, 1976; Esau and Charvat, 1978; Burgess and Linstead, 1984). These observations have indicated, during the cell death process in differentiating TEs, rapid and progressive degeneration of organelles, including the nucleus, vacuole, plastids, mitochondria, and the endoplasmic reticulum (ER), and finally the removal of protoplasts, the plasma membrane, and parts of primary walls that have not been lignified. Although shrinkage of nucleolar size, loss of nucleolar zonation, and, in some cases, clumping of chromatin occur during cell death of differentiating TEs (Lai and Srivastava, 1976), typical apoptosis-associated events such as shrinkage of the nucleus and cell and the formation of apoptotic bodies have never been found.

Recently Groover et al (1997) reported the detailed cell death process of differentiating TEs of Zinnia with electron microscope, epifluorescence microscope and time-lapse video. The visible degeneration of cellular organelles, including the nucleus, starts after the tonoplast disruption, which occurs several hours after secondary wall formation becomes visible. Their analysis with time-lapse video clearly indicates that the vacuole collapses inward in a few minutes. The differentiating TEs are cleaned of most contents within several hours after vacuole collapse. The nucleus is also degraded without lobbing, shrinkage or fragmentation. These observations imply that the disruption of the tonoplast is a critical step in programmed cell death and that the cytological features of this cell death process are not similar to those of apoptosis but rather to those of necrosis, although the cell death process of TEs is active. Another unique feature of cell death process during TE differentiation is that it occurs in cell-autonomous manner (Groover et al, 1997).

During the cell death process in TEs, ordered degradation of cell walls also occurs. The perforation of TEs is restricted to the ends of elongated cells. Burgess and Linstead (1984) reported that the middle lamella was resistant to autolysis when it was between neighboring cells in contact and only one differentiated, although the middle lamella between two neighboring TEs was digested completely. These results suggest that cell wall-hydrolytic enzymes are targeted into specific regions of cell walls and/ or that the modification of cell walls occurs at specific sites, probably before vacuolar collapse.

\section{Autolysis}

In apoptosis, DNA laddering is caused by DNA digestion in the nucleosome unit by endonucleases that are present in the nucleus, activated by $\mathrm{Ca}^{2+}$ and $\mathrm{Mg}^{2+}$ and inhibited by $\mathrm{Zn}^{2+}$ (Peitsh et al, 1993; Tanuma and Shiokawa, 1994). Mittler and Lam (1995a) indicated that a tobacco $36 \mathrm{kD}$ endonuclease, NUC III, which was expressed in association with cell death during the hypersensitive reaction caused by infection, has characteristics similar to apoptosis-related DNases, such as localization in the nucleus, $\mathrm{Ca}^{2+}$-activation, and $\mathrm{Zn}^{2+}$. inhibition. However, DNA laddering has not been observed under their hypersensitive conditions, and it is therefore still unknown whether NUC III can function in nuclear degradation in a manner similar to that of apoptosis-related endonucleases.

Thelen and Northcote (1989) found nuclease activities closely associated with TE differentiation in cultured Zinnia cells with in gel assay. These nucleases with molecular masses of $43 \mathrm{kD}, 22 \mathrm{kD}$ and $25 \mathrm{kD}$ appeared $12 \mathrm{~h}$ prior to the visible secondary wall thickenings of TEs, and their levels 
increased conspicuously during the autolytic stage. Among them only the $43 \mathrm{kD}$ nuclease had DNase activity that could hydrolyze both single-stranded and double-stranded DNA, although it could also hydrolyze RNA. The nuclease required $\mathrm{Zn}^{2+}$ for activation, which is unlike the apoptosis-related DNases. Such type of nucleases are also found to be expressed specifically in other cell death processes in higher plants, for example, in cell death in the aleurone during germination (Brown and Ho, 1986) and during leaf senescence (Blank and McKeon, 1989). This may imply that similar types of $\mathrm{Zn}^{2+}$-activated nucleases may be involved in different cell death processes in higher plants. Very recently, cDNA clones, BEN1 and ZEN1, that correspond to the $\mathrm{Zn}^{2+}$-activated nucleases were isolated from the barley aleurone and from differentiating Zinnia TEs, respectively (S Aoyagi, M Sugiyama and H Fukuda: BEN1 and ZEN1 cDNAs encoding S1-type DNases that are associated with programmed cell death in plants, unpublished observations). Their nucleotide and amino acid sequences showed that BEN1 and ZEN1 are similar to each other and to $\mathrm{S} 1$ nuclease of Aspergillus, indicating that the $\mathrm{S} 1$ type of nucleases with DNase activity may be commonly involved in nuclear degradation during different programmed cell death processes in plants. Furthermore BEN1 and ZEN1 proteins appeared to possess the signal peptide for targeting specific organelles or for secretion. However, because it has been indicated that the $43 \mathrm{kD}$ nuclease is not secreted (Thelen and Northcote, 1989), this nuclease may be localized in some intracellular compartment.

A histochemical analysis of fragmented DNA with TUNEL assay has revealed fragmentation of nuclear DNA in differentiating TEs in pea roots (Mittler and Lam, 1995b) and cultured Zinnia cells (Groover et al, 1997). However, this degradation of DNA in differentiating Zinnia TEs appears to occur near or after vacuole collapse (Groover et al, 1997). As judged from this result and many observations with electron and light microscopes, TUNELpositive signals in differentiating TEs do not seem to indicate apoptosis-like cell death, but rather result only from the high activity of DNases such as ZEN1 nuclease in differentiating TEs. However, it is still open to question whether this DNase reaches the nucleus and is activated before or after the vacuole collapse. The transcripts for ZRNase I, which may correspond to the $22 \mathrm{kD}$ (or $25 \mathrm{kD}$ ) RNase of Zinnia, are also induced specifically and transiently in differentiating TEs both in vitro and in situ (Ye and Droste, 1996).

Organellar degeneration is coupled with a high activity of proteases (Minami and Fukuda, 1995; Ye and Varner, 1996; Beers and Freeman, 1997). Single-cell protease assay has indicated that differentiating TEs themselves have high proteolytic activities, which suggests cellautonomous protein degradation in TEs (Beers and Freeman, 1997). Minami and Fukuda (1995) found that the Z-Phe-Arg-MCA hydrolytic activity showed a rapid increase just before the start of autolysis in Zinnia. This activity was derived from a TE-specific cysteine protease(s) with a $30 \mathrm{kD}$ molecular mass and a $\mathrm{pH}$ optimum of around $\mathrm{pH}$ 5. Our preliminary experiments with a membranepermeable inhibitor of cysteine proteases suggest that it suppresses the loss of the nucleus, and therefore that a cysteine protease(s) may be involved in nuclear degeneration (Yoriko Watanabe and Hiroo Fukuda, unpublished results). The autolysis-specific cysteine protease activity seems to result from the new induction of a cysteine protease gene(s) prior to the autolysis (p48h-17, Ye and Varner, 1993, 1996). The deduced amino acid sequence of this gene reveals high similarity to papain, and suggests the presence both of a presequence which directs the transport of the protein into some organelles or the secretion, and a prosequence whose removal may activate the protease. Recently ZCP4, which is similar to p48h-17 gene, has also been isolated from differentiating Zinnia cells, suggesting that at least two different species of cysteine protease genes are expressed in association with autolysis (Atsuishi Minami, Taku Demura and Hiroo Fukuda, unpublished results). In addition, a serine protease of $145 \mathrm{kD}$ was detected specifically in differentiating TEs on substrate-impregnated gels (Beers and Freeman, 1997). An approximately $60 \mathrm{kD}$ serine protease was also reported to appear preferentially in TE-inducing culture (Ye and Varner, 1996; Beers and Freeman, 1997). The activity of the $60 \mathrm{kD}$ protease is higher above $\mathrm{pH} 7$, which is unlike the $\mathrm{pH}$ optimum of the autolysis-specific cysteine protease, suggesting that at least two compartments with different $\mathrm{pHs}$ are involved in the autolysis. These results indicate that a complex set of proteases is involved in the autolytic process.

Transcripts for all of cell death-related genes examined, that is, p48h-17 gene, ZCP4, ZRNase I, and ZEN1 appear to accumulate transiently in a very similar pattern just before autolysis starts (Ye and Varner, 1993; Yamamoto et al, 1997; Ye and Droste, 1996; S Aoyagi, M Sugiyama and H Fukuda: BEN1 and ZEN1 cDNAs encoding S1-type DNases that are associated with programmed cell death in plants, unpublished observations). Therefore, the genes for these enzymes may be regulated to be expressed by a common mechanism, for example, by the same cis and trans activation factors. The deduced amino acid sequences of p48h-17 gene, ZCP4, ZRNase I, and ZEN1, taken together with our knowledge about the localization of hydrolytic enzymes in plants, indicate the presence of signal peptides, which allows us to speculate that these proteins are transported into the vacuole (Figure 1). Because the $\mathrm{pH}$ optima of the $43 \mathrm{kD}$ DNase (ZEN1 product) and the $30 \mathrm{kD}$ cysteine protease (possibly ZCP4 and/or p48h-17 product) are both 5-5.5, which corresponds to the $\mathrm{pH}$ in the vacuole, these hydrolytic enzymes may function in the vacuole or rather in the cytoplasm after the tonoplast disruption. The tonoplast disruption could permit hydrolytic enzymes to invade the cytoplasm and attack various organelles (Figure 1). Thus, the tonoplast disruption is a critical event in the cell death program of TEs. However, the mechanism of the tonoplast disruption is still open for investigation.

\section{Is there a common cell death mechanism?}

Recent extensive research on programmed cell death has revealed the presence of different types of cell death processes in plants. Infection and salt stress can induce 


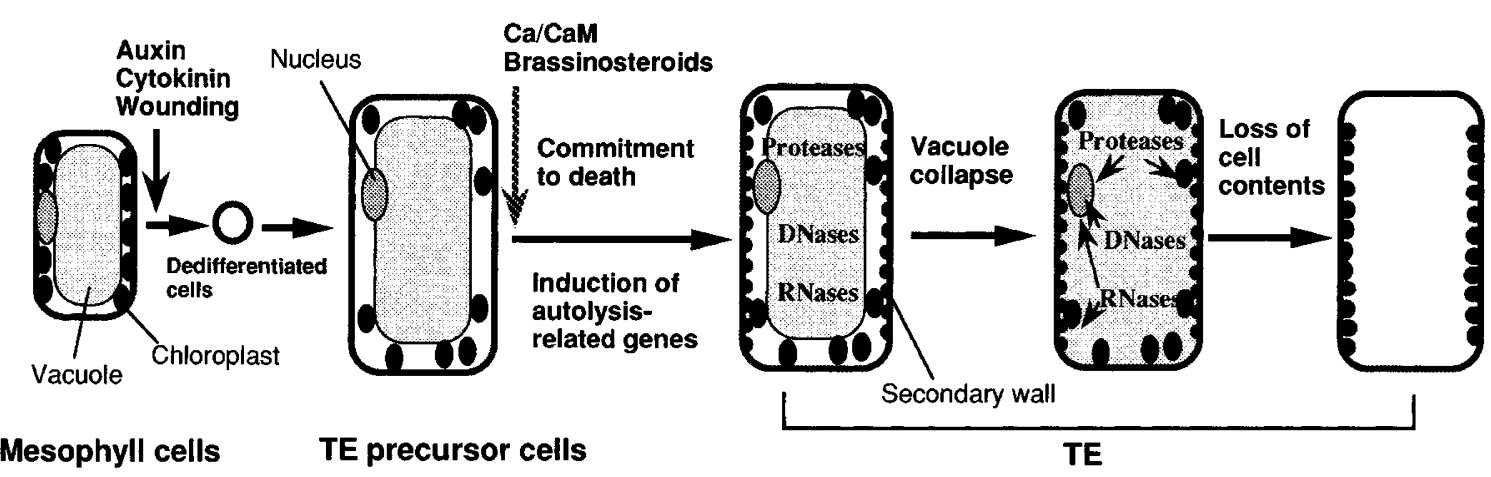

\begin{tabular}{|l|l|l|}
\hline Stage I & Stage II & Stage III \\
\hline
\end{tabular}

Figure 1 The process of cell death during TE differentiation. Mesophyll cells are induced to differentiate into TEs by wounding and a combination of auxin and cytokinin. The process of TE differentiation is divided into three stages, Stage I, II and III. The commitment to cell death may occur between Stage II and Stage III. After the commitment, autolysis-related genes such as genes for proteases, RNases, and DNases are induced. Their corresponding enzymes may be accumulated in the vacuole. The disruption of the tonoplast causes the hydrolytic enzymes to invade the cytoplasm and attack various organelles, resulting in the formation of a mature TE that has lost its cell contents and possesses decorated secondary walls

apoptosis-like cell death in plants (Wang et al, 1996a; Ryerson and Heath, 1996; Katsuhara and Kawasaki, 1996). On the other hand, infection can also cause necrotic death in plant cells (Mittler and Lam, 1995a). Even among developmentally programmed cell death processes, there seem to be different types. Wang et al (1996a) indicated that cells died in an apoptosis-like manner during development in onion root caps. In contrast, cell death processes in TE differentiation as mentioned above and in senescence of leaves (Smart, 1994) and ovary (Vercher et al, 1987) are nearer to necrosis than apoptosis. Is there a mechanism common to programmed cell death processes in plants? At present, there are no data supporting a common death-signal(s) that directly induces developmentally regulated cell death in plants. In senescence of leaves, swelling degradation of organelles occurs (Smart, 1994). This is similar to the autolytic process of TE differentiation. In leaf senescence, however, chloroplasts are gradually degraded before the vacuolar collapse, which is conspicuously different from TE differentiation. In contrast, the tonoplast disruption is a critical factor of the death of endocarp cells during ovary senescence of pea (Vercher et al, 1987). Aleurone cells die in response to GA (Kuo et al, 1996). This cell death process involves vacuolar enlargement and then the disruption of the tonoplast and plasma membrane, although it is unknown whether the vacuolar collapse precedes that of the plasma membrane. Thus, during development of plants, there is one common type of cell death process in which a key event is active vacuolar collapse. Interestingly, similar hydrolytic enzymes are commonly expressed during this type of cell death. During pea ovary senescence, a cysteine protease gene, tpp is expressed preferentially in endocarp and ovule as well as vascular bundles, in which tpp is expressed even when the ovary is non-senescent (Granell et al, 1992). This gene is highly homologous to $448 \mathrm{~h}-17$ gene and ZCP4. Genes for a similar type of cysteine proteases are also expressed in aleurone of barley (Rogers et al, 1985) and in rice (Watanabe et al, 1991). In addition, the S1 type nucleases are expressed commonly in cell death processes of the barley aleurone and Zinnia TEs (S Aoyagi, M Sugiyama and H Fukuda: BEN1 and ZEN1 cDNAs encoding S1-type DNases that are associated with programmed cell death in plants, unpublished observations). Thus, there may at least be some common cell death mechanism among the different developmental processes in which the vacuolar collapse is a critical event in cell death.

\section{Conclusion}

Intensive cellular and molecular analyses have dissected the programmed cell death process of TEs and have indicated that the process is unique in that the vacuolar collapse is a critical step of cell death. However, the data that have been obtained so far are still fragmentary and many important questions remain unresolved: What is the cell death signal(s) and when do differentiating cells perceive the signal(s)? Brassinosteroids may be one of its candidates. Is cell death signal(s) endogenous or supplied by neighboring cells? How are signals transduced in cells from the perception of the cell death signal to the expression of cell death genes? The vacuole collapse is a critical irreversible point of cell death. What is the signal for the vacuole collapse? After the vacuole collapses, organelles are degraded rapidly by hydrolytic enzymes. Are there a common mechanism for expression of these hydrolytic enzymes and their targeting into specific organelles? Cell death and secondary wall formation in TE differentiation seem to be tightly coupled, in particular on gene expression. Do the two processes share a regulation mechanism of gene expression? To answer these questions, we need to isolate key molecules that function in specific steps of cell death. The Zinnia system will be useful for such a purpose, because of easy following of cell death process and high synchrony of cell death process. In addition, 
we will have to develop cellular experimental systems in which functions of genes and proteins in cell death are easily tested. Meanwhile the analyses of cell death process during TE differentiation, its components and the signals to this unique cell death are providing a useful clue to the mechanism of general cell death program in plants.

\section{Acknowledgements}

Financial support from the Ministry of Education, Science and Culture of Japan (No. 05276103, No. 06278102, No. 07454215), the Japan Society for the Promotion of Science (JSPS-RFTF96L00605) is gratefully acknowledged.

\section{References}

Beers EP and Freeman TB (1997) Protease activity during tracheary element differentiation in Zinnia mesophyll cultures. Plant Physiol. 113: 873-880

Blank A and McKeon TA (1989) Single-strand-preferring nuclease activity in wheat leaves is increased in senescence and is negatively photoregulated. Proc. Natl. Acad. Sci. USA. 86: 3169-3173

Brown PH and Ho THD (1986) Barley (Hordeum vulgare cultivar Himalaya) aleurone layers secrete a nuclease in response to gibberellic acid: Purification and partial characterization of the associated RNase, DNase, and 3 -nucleotidase activities. Plant Physiol. 82: 801-806

Burgess J and Linstead P (1984) In vitro tracheary element formation: Structural studies and the effect of triiodobenzoic acid. Planta 160: 481-489

Chasan R (1994) Tracing tracheary element development. Plant Cell. 6: 917-919

Demura T and Fukuda H (1993) Molecular cloning and characterization of cDNAs associated with tracheary element differentiation in cultured Zinnia cells. Plant Physiol. 103: 815-821

Demura Tand Fukuda H(1994) Novel vascular cell-specific genes whose expression is regulated temporally and spatially during vascular system development. Plant Cell 6: $967-981$

Esau Kand CharvatI(1978) On vessel member differentiation in the bean (Phaseolus vulgaris L.). Ann Bot. 42: 665-677

Fukuda H (1994) Redifferentiation of single mesophyll cells into tracheary elements. Int. J. Plant Sci. 155: 262-271

Fukuda H (1996) Xylogenesis: Initiation, progression and cell death. Ann. Rev. Plant Physiol. Plant Mol. Biol. 47: 299-325

Fukuda H (1997) Tracheary element differentiation. Plant Cell. 9: 1147-1156

Fukuda $\mathrm{H}$ and Komamine A (1980a) Establishment of an experimental system for the tracheary element differentiation from single cells isolated from the mesophyll of Zinnia elegans. Plant Physiol. 65: 57-60

Fukuda $\mathrm{H}$ and Komamine A (1980b) Direct evidence for cytodifferentiation to tracheary elements without intervening mitosis in a culture of single cells isolated from the mesophyll of Zinnia elegans. Plant Physiol. 65: 61-64

Fukuda $\mathrm{H}$ and Komamine A (1983) Changes in the synthesis of RNA and protein during tracheary element differentiation in single cells isolated from the mesophyll of Zinnia elegans. Plant Cell Physiol. 24: 603-614

Granell A, Harris N, Pisabarro AG and Carbonell J (1992) Temporal and spatia expression of a thiolprotease gene during pea ovary senescence, and its regulation by gibberellin. Plant J. 2: 907-915

Groover A, DeWitt N, Heidel A and Jones A (1997) Programmed cell death of plant tracheary elements differentiating in vitro. Protoplasma 196: 197-211

Iwasaki T and Shibaoka H (1991) Brassinosteroids act as regulators of trachearyelement differentiation in isolated Zinnia mesophyll cells. Plant Cell Physiol. 32: $1007-1014$

Jones AM and Dangl J (1996) Logjam at the Styx: Programmed cell death in plants. Trends in Plant Sci. 1: 114-119

Katsuhara K and Kawasaki T (1996) Salt stress induced nuclear and DNA degradation in meristematic cells of barley roots. PlantCell Physiol. 37: 169-173

Kerr JFR and Harmon BV (1991) Definition and incidence of apoptosis: An historical perspective. In Apoptosis: The Molecular Basis of Cell Death. LD Tomei and FO Cope eds. (New York: Cold Spring Harbor Lab. Press), pp. 5-29
Kobayashi $\mathrm{H}$ and Fukuda $\mathrm{H}$ (1994) Involvement of calmodulin and calmodulinbinding proteins in the differentiation of tracheary elements in Zinnia cells. Planta 194: $388-394$

Kobayashi H, Fukuda H and Shibaoka H (1987) Reorganization of actin filaments associated with the differentiation of tracheary elements in Zinnia mesophyll cells. Protoplasma 138: $69-71$

Kuo A, Cappelluti S, Cervantes-Cervantes M, Rodriguez M and Bush DS (1996) Okadaic acid, a protein phosphatase inhibitor, blocks calcium changes, gene expression, and cell death induced by gibberellin in wheat aleurone cells. Plant Cell. 8: 259-269

Lai V and Srivastava LM (1976) Nuclear changes during differentiation of xylem vessel elements. Cytobiologie 12: 220-243

Minami A and Fukuda H (1995) Transient and specific expression of a cysteine endopeptidase during autolysis in differentiating tracheary elements from Zinnia mesophyll cells. Plant Cell Physiol. 36: 1599-1606

Mittler R and Lam E (1995a) Identification, characterization, and purification of a tobacco endonuclease activity induced upon hypersensitive response cell death. Plant Cell. 7: 1951-1962

Mittler R and Lam E (1995b) In situ detection of nDNA fragmentation during the differentiation of tracheary elements in higher plants. Plant Physiol. 108: 489493

O'Brien TP and Thimann KV (1967) Observation on the fine structure of the oat coleoptile III. Correlated light and electron microscopy of the vascular tissues. Protoplasma 63: 443-478

Peitsh MC, Polzar B, Stepham H, Crompton T, MacDonald HR, Mannherz HG and Tshopp J (1993) Characterization of the endogenous deoxyribonuclease involved in nuclear DNA degradation during apoptosis (programmed cell death). EMBO J. 12: 371-377

Roberts AW and Haigler CH (1989) Rise in chlortetracycline accompanies tracheary element differentiation in suspension cultures of Zinnia. Protoplasma 152: 37

Rogers JC, Dean D and Heck GR (1985) Aleurain: A barley thiol protease closely related to mammalian cathepsin H. Proc. Natl. Acad. Sci. USA. 82: 6512-6516

Ryerson DE and Heath MC (1996) Cleavage of nuclear DNA into oligonucleosomal fragments during cell death induced by fungal infection or by abiotic treatments. Plant Cell 8: 393-402

Smart CM (1994) Gene expression during leaf senescence. New Phytol. 126: 419 448

Srivastava LM and Singh AP (1972) Certain aspects of xylem differentiation in corn. Can J. Bot. 50: 1795-1804

Tanuma SI and Shiokawa D (1994) Multiple forms of nuclear deoxyribonuclease in rat thymocytes. Biochem. Biophys. Res. Com. 203: 789-797

Thelen MP and Northcote DH (1989) Identification and purification of a nuclease from Zinnia elegans L.: A potential molecular marker for xylogenesis. Planta 179: $181-195$

Vercher Y, Molowny A and Carbonell J (1987) Gibberellic acid effects on the ultrastructure of endocarp cells of unpollinated ovaries of Pisum sativum. Physiol. Plant. 71: 302-308

Wang H, Li J, Bostock RM and GilchristDG (1996a) Apoptosis: A functional paradigm for programmed plant cell death induced by a host-selective phytotoxin and invoked during development. Plant Cell. 8: 375-391

Wang M, Oppedijk BJ, Lu X, Duijn BV and Schilperoort RA (1996b) Apoptosis in barley aleurone during germination and its inhibition by abscisic acid. Plant Mol. Biol. 32: 1125-1134

Watanabe H, Abe K, Emori Y, Hosoyama H and Arai S (1991) Molecular cloning and gibberellin-induced expression of multiple cysteine proteases of rice seeds (Orizains). J. Biol. Chem. 266: 16897-16907

Yamamoto R, Demura T and Fukuda H (1997) Brassinosteroids induce entry into the final stage of tracheary element differentiation in cultured Zinnia cells. Plant and Cell Physiol. 38: 980-983

Ye Z-H and Droste DL (1996) Isolation and characterization of cDNAs encoding xylogenesis-associated and wound-induced ribonucleases in Zinnia elegans. Plant Mol. Biol. 30: 697-709

Ye Z-H and Varner JE (1993) Gene expression patterns associated with in vitro tracheary element formation from isolated mesophyll cells of Zinnia elegans. Plant Physiol. 103: 805-813

Ye Z-H and Varner JE (1996) Induction of cysteine and serine proteases during xylogenesis in Zinnia elegans. Plant Mol. Biol. 30: 1233-1246 